TRANSACTIONS OF THE

AMERICAN MATHEMATICAL SOCIETY

Volume 195, 1974

\title{
$T$-FAITHFUL SUBCATEGORIES AND LOCALIZATION
}

BY

JOHN A. BEACHY

ABSTRACT. For any additive functor from a category of modules into an abelian category there is a largest Giraud subcategory for which the functor acts faithfully on homomorphisms into the subcategory. It is the largest Giraud subcategory into which the functor reflects exact sequences, and under certain conditions it is just the largest Giraud subcategory on which the functor acts faithfully. If the functor is exact and has a right adjoint, then the subcategory is equivalent to the quotient category determined by the kernel of the functor. In certain cases, the construction can be applied to a Morita context in order to obtain a recent theorem of Mueller.

Similarly, the functor defines a certain reflective subcategory and an associated radical, which is a torsion radical in case the functor preserves monomorphisms. Certain results concerning this radical, when defined by an adjoint functor, can be applied to obtain two theorems of Morita on balanced modules.

This paper is a continuation of the author's work [2] on $T$-faithful subcategories of module categories. For the category ${ }_{R}$ M of unital left $R$-modules over a ring $R$ with identity, and for any additive functor $T:{ }_{R} \mathbb{M} \rightarrow \mathbb{B}$ into an abelian category $\mathfrak{B}$, a subcategory $\mathbb{Q}$ of ${ }_{R} \mathbb{M}$ will be called $T$-faithful if $T$ is faithful when restricted to $\mathbb{Q}$. (Similarly, $\mathbb{Q}$ is left $T$-faithful if $T(f) \neq 0$ for every nonzero $R$-homomorphism $f: M \rightarrow A$, with $A \in \mathbb{Q}$.) If $T$ is exact and has an adjoint, one can form the quotient category ${ }_{R} \mathbb{M} / \operatorname{Ker}(T)$, which is then equivalent to a certain Giraud subcategory of $R$. This Giraud subcategory can be characterized as the largest left $T$-faithful Giraud subcategory, and if $R$ is commutative and Noetherian, it is the largest $T$-faithful Giraud subcategory of ${ }_{R}$ M (Corollary 3.10).

For any functor $T:{ }_{R} M \rightarrow \mathscr{B}$ it is possible to construct the largest Giraud subcategory of ${ }_{R}$ M which is left $T$-faithful, thus generalizing in a certain sense the construction of the quotient category ${ }_{R} \mathbb{M} / \operatorname{Ker}(T)$. If $T$ is exact, this subcategory is determined by the smallest hereditary torsion class of $R_{R}$ /N which contains $\operatorname{Ker}(T)$. The construction is useful even when $T$ is not exact. Mueller

Received by the editors April 2, 1973.

AMS (MOS) subject classifications (1970). Primary 16A08; Secondary 18E35, 18E40.

Key words and phrases. T-faithful subcategory, torsion radical, quotient category, balanced module.

Copyright $\odot$ 1974, American Mathematical Society 
has shown in [12] that any Morita context induces an equivalence between appropriate quotient categories. In certain cases these subcategories are precisely the ones constructed in the above manner.

$\$ 1$ studies reflective $T$-faithful subcategories, and gives the construction of a subcategory $\mathfrak{L F}(T)$ which is the largest left $T$-faithful reflective subcategory of ${ }_{R}$ M. This determines a radical $\operatorname{rad}_{T}$ of ${ }_{R}$, which is a torsion radical if $T$ is a monofunctor (Theorem 1.8). A characterization of $\operatorname{rad}_{T}$ is given when $T$ has a right adjoint. $\$ 2$ gives some examples, and shows that if $T=\operatorname{Hom}_{R}(P,-)$ for a module ${ }_{R} P$, then $\operatorname{rad}_{T}$ is determined by the trace ideal of $P$. In this case conditions are given under which the torsion class determined by $\operatorname{rad}_{T}$ is a TTF-class.

$\$ 3$ is concerned with the construction of a Giraud subcategory determined by $\operatorname{rad}_{T}$. This is characterized in Theorem 3.7 as the largest Giraud subcategory $\mathbb{Q}$ (with exact reflector $Q:{ }_{R} \mathbb{N} \rightarrow \mathbb{Q}$ ) which satisfies any one of the following conditions: (i) $\mathbb{Q}$ is $T$-faithful and $T(f)=0$ implies $T Q(f)=0$ for all $R$-homomorphisms f; (ii) $T(f)=0$ implies $Q(f)=0$ for all $R$-homomorphisms $f$; (iii) for any sequence $M^{\prime} \rightarrow M \rightarrow M^{\prime \prime}$ in $R$, the image of the sequence under $Q$ is exact if the image of the sequence under $T$ is exact. Thus one might loosely say that the construction gives the largest Giraud subcategory into which $T$ reflects exact sequences, generalizing the statement that $T$ reflects exact sequences if it is faithful.

$\$ 4$ gives an application to Morita contexts, and also generalizes several theorems of Morita [11]. If $T$ is covariant and has a right adjoint $S$ or is contravariant with $S$ adjoint on the right, then for each module $M \in \epsilon_{R} M, \operatorname{rad}_{T}$ determines a module $Q_{T}(M) \in \mathscr{L F}(T)$ which is analogous to a module of quotients, and coincides with the usual module of quotients if $T$ is exact. Theorem 4.9 shows that the homomorphism $M \rightarrow S T(M)$ induced by the adjoint situation is an isomorphism if and only if $Q_{T}(M)=S T(M)$ and there exists an exact sequence $0 \rightarrow M \rightarrow$ $S(B) \rightarrow N$, for some $B \in \mathcal{B}$ and $N \in \mathfrak{L F}(T)$. As an application of the theorem, it is shown that a faithful module ${ }_{R} V$ is balanced if and only if $V$-dom $\operatorname{dim}{ }_{R} R \geq 2$ and $V$ is a module of type $F_{b}$, a result due to Morita [11, Theorem 5.1].

The results have been stated for categories of modules, since the examples and applications of interest to the author are found in module categories, but the proofs can be extended immediately. The construction of $\mathscr{L F}(T)$ in $\$ 1$ requires an abelian category which is complete and locally small. The construction of $\mathcal{L F}_{2}(T)$ in $\S_{3}$ requires in addition the existence of enough injectives.

1. Reflective $T$-faithful subcategories. In this section $T:{ }_{R} M \rightarrow \mathscr{B}$ will denote an additive functor (either covariant or contravariant) into an abelian category $B$. The full subcategory of ${ }_{R} M$ determined by those modules ${ }_{R} M$ such 
that $T(f) \neq 0$ for all $R$-homomorphisms $0 \neq f:{ }_{R} X \rightarrow{ }_{R} M$ will be denoted by $\mathscr{L F}(T)$. (See [2].) Recall that if $T$ is covariant, then a covariant functor $S: B \rightarrow{ }_{R} \mathbb{M}$ is a right adjoint for $T$ (and $T$ is a left adjoint for $S$ ) if there exists a natural equivalence of set-valued bifunctors $\eta: \operatorname{Hom}_{B}(T(\cdot),-) \rightarrow \operatorname{Hom}_{R}(\cdot, S(-))$. For any $M \in \epsilon_{R} M$ and $B \in \mathscr{B}$, the natural equivalence induces morphisms $\phi_{M}=$ $\eta\left(1_{T(M)}\right): M \rightarrow S T(M)$ and $\psi_{B}=\eta^{-1}\left(1_{S(B)}\right): T S(B) \rightarrow B$, and for any morphism $f: M \rightarrow S(B), \eta^{-1}(f)$ is the unique $R$-homomorphism for which the following diagram commutes

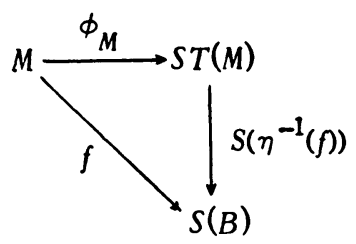

Furthermore, $\eta^{-1}(f)=\psi_{B} T(f)$, and so $f \neq 0$ implies $T(f) \neq 0$, which shows that $S(B)$ belongs to $\mathfrak{F F}(T)$.

If $T$ is covariant and has a right adjoint, then the dual of [2, Proposition 1.7] shows that, for any $M \in \epsilon_{R} \mathbb{M}, M \in \mathscr{L F}(T)$ if and only if $\phi_{M}$ is a monomorphism. Note that $\mathfrak{L F}(T)=\mathscr{L F}(S T)$. It is immediate that $\mathfrak{W}(S T) \subseteq \mathscr{W}(T)$, and on the other hand, if $M \in \mathfrak{L F}(T)$, then $\phi_{M}: M \rightarrow S T(M)$ is a monomorphism, so $\phi_{M} f \neq 0$ for any $R$-homomorphism $0 \neq f: X \rightarrow M$. But then $S T(f) \phi_{S T(X)} \neq 0$ and this implies $S T(f) \neq 0$.

A subcategory $\mathbb{Q}$ of ${ }_{R} M$ is called a reflective subcategory if the inclusion functor Inc: $\mathbb{Q} \rightarrow{ }_{R} \mathbb{M}$ has a left adjoint (or reflector) $Q:{ }_{R} \mathbb{M} \rightarrow \mathbb{Q}$. That $Q$ is a left adjoint is equivalent to the existence, for each $M \in \epsilon_{R}$, of an $R$-homomorphism $\phi_{M}: M \rightarrow Q(M)$ such that any $R$-homomorphism $f: M \rightarrow A$, with $A \in \mathbb{Q}$, factors uniquely through $\phi_{M}$. (Here $A$ and Inc $(A)$ have been identified.) The subcategory $\mathscr{Q}$ is called epireflective if $\phi_{M}$ is an epimorphism for each $M \epsilon_{R} \mathbb{M}$. In this case $Q$ preserves epimorphisms.

Definition (1.1) Let $T:{ }_{R} \mathbb{M} \rightarrow \mathfrak{B}$ be a functor. For each $M \epsilon_{R} \mathbb{M}$, define $\operatorname{rad}_{T}(M)=\left\{m \in M: f(m)=0\right.$ for all $f \in \operatorname{Hom}_{R}(M, X)$ such that $\left.X \in \mathfrak{L F}(T)\right\}$.

It can be checked easily that $\operatorname{rad}_{T}$ defines a radical of ${ }_{R} \mathbb{N}$, that is, that, for all $R$-homomorphisms $f:{ }_{R} M \rightarrow{ }_{R} N, f\left(\operatorname{rad}_{T}(M)\right) \subseteq \operatorname{rad}_{T}(N)$, and moreover, that $\operatorname{rad}_{T}\left(M / \operatorname{rad}_{T}(M)\right)=0$ for all $M \epsilon_{R}$ M. Recall that a left exact radical is called a torsion radical. Theorem 1.8 gives conditions under which $\operatorname{rad}_{T}$ is a torsion radical. If $T$ is covariant and has a right adjoint, then for all $M \in \epsilon_{R} M, \operatorname{rad}_{T}(M)=$ $\operatorname{ker}\left(\phi_{M}\right)$. That $\operatorname{rad}_{T}(M) \subseteq \operatorname{ker}\left(\phi_{M}\right)$ follows since $\phi_{M}: M . S T(M)$ and $S T(M) \in \mathscr{L} \mathcal{F}(T)$. On the other hand, any $R$-homomorphism $f: M \rightarrow{ }_{R} X$ yields the following commutative diagram. 


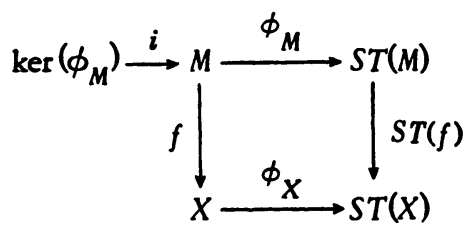

If $X \in \mathfrak{L F}(T)$, then $\phi_{X}$ is a monomorphism, and so $S T(f) \phi_{M} i=0$ implies $\phi_{X} f i=0$ and therefore $f i=0$. Thus $\operatorname{ker}\left(\phi_{M}\right) \subseteq \operatorname{rad}_{T}(M)$.

Proposition (1.2) For any functor $T:{ }_{R} \mathbb{M} \rightarrow \mathfrak{B}, \mathfrak{L F}(T)$ is an epireflective subcategory, and $\mathscr{L F}(T)=\mathscr{L F}(Q)$ for the reflector $Q:{ }_{R} \mathbb{M} \rightarrow \mathfrak{L F}(T)$.

Proof. Since $\mathfrak{L F}(T)$ is complete [2, Corollary 1.6] and closed under submodules, it is epireflective [9, Chapter V, Proposition 6.1]. For $M \in_{R} \mathbb{M}$, $Q(M)=M / \mathrm{rad}_{T}(M)$ defines the reflector $Q$, and by the preceding remarks $M \in \mathfrak{L F}(Q) \Leftrightarrow \operatorname{rad}_{T}(M)=0 \Leftrightarrow M \in \mathscr{\mathscr { F }}(T)$.

Lemma (1.3) Let $\mathbb{Q}$ be a reflective subcategory of $R_{R}$ II with reflector $Q$, and let $T:{ }_{R} \mathbb{M} \rightarrow \mathfrak{B}$. If $M \in \in_{R} \mathbb{M}$ and $Q(M) \in \mathscr{L} \mathcal{F}(T)$, then $T(f)=0$ implies $Q(f)=0$, for any $R$-bomomorphism $f:{ }_{R} X \rightarrow{ }_{R} M$.

Proof. If $T(f)=0$, then $T\left(\phi_{M} f\right)=0$, and since $Q(M) \in \mathscr{L F}(T)$ this implies that $\phi_{M} f=0$. But then $Q(f)=0$ since $Q(f)$ is the unique homomorphism which makes the following diagram cornmutative.

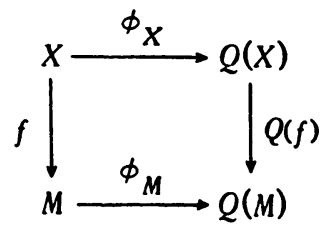

Theorem (1.4) Let $\mathbb{Q}$ be a reflective subcategory of $R^{M}$ with reflector $Q$, and let $T:{ }_{R} \mathbb{M} \rightarrow \Re$ be any functor. Then the following conditions are equivalent:

(a) $\mathbb{Q}$ is a subcategory of $\mathscr{L F}(T)$.

(b) $\mathbb{Q}$ is $T$-faithful, and $T(f)=0$ implies $T Q(f)=0$, for all $R$-bomomorpbisms $f$.

(c) $T(f)=0$ implies $Q(f)=0$, for all R-homomorphsims $f$.

Proof. (a) $\Rightarrow$ (b). If $\mathfrak{Q}$ is a subcategory of $\mathfrak{L F}(T)$, then $\mathfrak{Q}$ is $T$-faithful since $\mathscr{L F}(T)$ is $T$-faithful. For any $R$-homomorphism $f:{ }_{R} X \rightarrow{ }_{R} M, Q(M) \in \mathfrak{L F}(T)$ and so by Lemma 1.3, $T(f)=0$ implies $Q(f)=0$ and hence $T Q(f)=0$.

(b) $\Rightarrow$ (c). If $T(f)=0$, then by assumption $T Q(f)=0$. But $Q(f)$ is in $Q$ and $\mathbb{Q}$ is $T$-faithful, so $Q(f) \doteq 0$.

(c) $\Rightarrow$ (a). If $T(f)=0$ implies $Q(f)=0$, then $\mathscr{L F}(Q) \subseteq \mathscr{L F}(T)$. The conclusion follows from previous remarks about adjoints, since the inclusion functor is a 
right adjoint for $Q$, and hence $A \in \mathfrak{L F}(Q)$ for all $A \in \mathbb{Q}$.

This shows that $\mathscr{L F}(T)$ is the largest reflective $T$-faithful subcategory whose reflector $Q$ satisfies the condition $T(f)=0$ implies $T Q(f)=0$. The next corollaries are attempts to remove the last condition. A functor will be called an epifunctor if it is covariant and preserves epimorphisms or contravariant and carries epimorphisms to monomorphisms.

Corollary (1.5) Let $\mathcal{Q}$ be an epireflective subcategory of $R_{R} \mathbb{M}$, and let $T:{ }_{R} \mathbb{M} \rightarrow \mathfrak{B}$ be a functor, with reflector $Q:{ }_{R} \pi \rightarrow \mathfrak{W F}(T)$.

(a) If $T$ is an epifunctor, then $\mathbb{Q}$ is $T$-faithful $\Leftrightarrow \mathbb{Q} \subseteq \mathfrak{Q F}(T)$.

(b) $\mathbb{Q}$ is $Q$-faithful $\Leftrightarrow \mathbb{Q} \subseteq \mathfrak{Q F}(T)$.

Proof. (a) Assume that $Q$ is $T$-faithful, with reflector $Q_{0}:{ }_{R} \pi \rightarrow Q$, and that $T$ is a covariant epifunctor. If $f \in \operatorname{Hom}_{R}(M, N)$ and $T(f)=0$, then $T Q(f) T\left(\phi_{M}\right)=T\left(\phi_{N}\right) T(f)=0$, so $T Q(f)=0$ since by assumption $T\left(\phi_{M}\right)$ is an epimorphism. By Theorem 1.4(b), $Q \subseteq \mathfrak{L F}(T)$.

(b) This follows from (a), since $Q$ is an epifunctor and $\mathscr{L F}(Q)=\mathscr{L F}(T)$.

Corollary (1.6) If $T:{ }_{R} \mathbb{R} \rightarrow \mathscr{B}$ is an epifunctor, then $\mathscr{\mathscr { F }}(T)$ is the largest epireflective T-faitbful subcategory of $R^{M}$.

Corollary (1.7) Let $\mathbb{Q}$ be a reflective subcategory of $R^{\Uparrow \text {, with reflector }}$ $T:{ }_{R} \pi \rightarrow \mathbb{Q}$. Then $T$ is naturally isomorpbic to $T Q$, for the reflector $Q:{ }_{R} \pi \rightarrow$ $\mathfrak{L F}(T)$, and for all $M \in_{R} \mathbb{M}, M \rightarrow Q(M) \rightarrow T Q(M)$ is the factorization of $M \rightarrow T(M)$ into an epimorphism followed by a monomorphism.

Proof. For any $M \epsilon_{R} M, M \rightarrow Q(M)$ is an epimorphism, and $Q(M) \rightarrow T Q(M)$ is a monomorphism since $Q(M) \in \mathfrak{L F}(T)$. If $A \in \mathbb{Q}$ and $f: M \rightarrow A$, then $f$ factors uniquely through $Q(M)$ since $A \in \mathfrak{L F}(T)$. But then $f$ further factors uniquely through $T Q(M)$, and so this shows that $T Q$ is a left adjoint for the inclusion functor. Since adjoints are unique up to natural isomorphism, $T Q$ is naturally isomorphic to $T$.

A class $\mathcal{C}$ of modules is called a torsion class if it is closed under formation of homomorphic images, direct sums, and (group) extensions, and a torsionfree class if it is closed under submodules, direct products, and (group) extensions. A torsion class is called hereditary if it is closed under submodules. There is a one-to-one correspondence between hereditary torsion classes, torsion radicals, and torsionfree classes closed under essential extensions [13, Proposition 2.6].

Theorem (1.8) Let $T:{ }_{R} \mathbb{M} \rightarrow \mathbb{B}$ be a functor.

(a) If $T$ is a monofunctor, then $\operatorname{rad}_{T}$ is a torsion radical.

(b) If $T$ is exact, then the torsion class of $\operatorname{rad}_{T}$ is the smallest bereditary torsion class containing $\mathrm{Ker}(T)$. 
Proof. (a) If $T$ is a monofunctor, then by the dual of [2, Proposition 2.4], $\mathfrak{L F}(T)$ is closed under (group) extensions, and by [2, Proposition 2.5], it is closed under essential extensions. Therefore the $\operatorname{radical}^{\operatorname{rad}_{T}}$ determined by $\mathfrak{L F}(T)$ is a torsion radical.

(b) By [2, Proposition 2.6], $M \in \mathfrak{L F}(T)$ if and only if $\operatorname{Hom}_{R}(K, M)=0$ for all $K \in \operatorname{Ker}(T)$. The torsion class of $\operatorname{rad}_{T}$ is the collection of modules ${ }_{R} N$ such that $\operatorname{Hom}_{R}(N, M)=0$ for all $M$ in $\mathcal{L F}(T)$, and so this is precisely the hereditary torsion class generated by $\operatorname{Ker}(T)$.

2. Examples. For modules $R_{R}^{M}$ and ${ }_{R} P$, the functors $\operatorname{Hom}_{R}(-, M)$ and $\operatorname{Hom}_{R}(P,-)$ will be denoted by $H_{M}$ and $H^{P}$ respectively. In [2], $\mathscr{L F}\left(H_{M}\right)$ and $\mathfrak{R F}\left(H^{P}\right),\left(R \mathcal{F}\left(H^{P}\right)\right.$ is defined dually) were studied. Every torsion radical has the form $\operatorname{rad}_{T}$ for $T=H_{M}$ for an appropriate injective module ${ }_{R} M$, since $\mathscr{Q F}(T)$ is just the full subcategory of modules cogenerated by $M$. The main purpose of this section is to study $\mathfrak{L F}\left(H^{P}\right)$, which by Theorem 1.8 determines a torsion radical since $H^{P}$ is always left exact.

For modules $R_{R}^{M}$ and ${ }_{R} P$, let $\operatorname{tr}_{M}(P)$ be the sum in $M$ of all $R$-homomorphic images of $P$. An ideal $A$ of $R$ will be called a trace ideal if $A=\operatorname{tr}_{R}(P)$ for some module ${ }_{R} P$. It is evident that $A$ is a trace ideal if and only if $A=\operatorname{tr}_{R}(A)$, and that trace ideals are just the torsion ideals of idempotent preradicals. In particular, any idempotent ideal is a trace ideal. (See [3].)

Proposition (2.1) Let $P \in \in_{R}$ M, and let ${ }_{R} U$ be a generator for ${ }_{R}$ M.

(a) $\mathfrak{L F}\left(H^{P}\right)=\mathfrak{L F}\left(H^{V}\right)$, for ${ }_{R} V=\operatorname{tr}_{U}(P)$.

(b) $M \in \mathfrak{L F}\left(H^{P}\right) \Leftrightarrow \operatorname{Hom}_{R}(U / V, M)=0$.

Proof. (a) Since $P$ generates $V, H^{V}(f) \neq 0$ implies $H^{P}(f) \neq 0$, for any $R$ homomorphism $f$, and so $\mathscr{L F}\left(H^{V}\right) \subseteq \mathfrak{Q F}\left(H^{P}\right)$. On the other hand, if $M \in \mathscr{\mathscr { F }}\left(H^{P}\right)$ and $0 \neq f:{ }_{R} X \rightarrow{ }_{R} M$, then since $U$ is a generator there exists a homomorphism $b: U \rightarrow X$ with $f b \neq 0$. Since $H^{P}(f b) \neq 0$ by assumption, there exists $g: P \rightarrow U$ with $f h g \neq 0$, and then $f b(V) \neq 0$ because $g(P) \subseteq V$. Thus $H^{V}(f) \neq 0$, and $M \in \mathscr{L F}\left(H^{V}\right)$.

(b) $\left(\Rightarrow\right.$. If $M \in \mathfrak{L F}\left(H^{P}\right)$ and $\operatorname{Hom}_{R}(U / V, M) \neq 0$, say $0 \neq f: U / V \rightarrow M$, then $H^{P}(f p) \neq 0$ for the projection $p: U \rightarrow U / V$, so there exists $g: P \rightarrow U$ with $f p g \neq 0$. But $g(P) \subseteq V$ since $V=\operatorname{tr}_{U}(P)$, a contradiction.

$\Leftrightarrow)$. If $0 \neq f: X \rightarrow M$, then there exists $g: U \rightarrow X$ with $f g \neq 0$, since $U$ is a generator. But then if $\operatorname{Hom}_{R}(U / V, M)=0, f g(V) \neq 0$, since otherwise $f g$ would factor through $U / V$. Therefore $M \in \mathfrak{L F}\left(H^{V}\right)=\mathfrak{L F}\left(H^{P}\right)$.

Corollary (2.2) Let $P \in{ }_{R} M$ and $A=\operatorname{tr}_{R}(P)$. Then $M \in \mathscr{L F}\left(H^{P}\right) \Leftrightarrow$ $\operatorname{Ann}_{M}(A)=0$. 
Proof. By Proposition 2.1, $M \in \mathfrak{L F}\left(H^{P}\right) \Leftrightarrow \operatorname{Hom}_{R}(R / A, M)=0$, and this occurs if and only if $\mathrm{Ann}_{M}(A)=\{m \in M: A m=0\}=0$.

Corollary (2.3) Let $P \in \in_{R} M$, and let $T=H^{P}$. Then $\operatorname{rad}_{T}$ is the identity functor on $R^{M}$ if and only if $\operatorname{tr}_{R}(P)$ is a right t-nilpotent ideal.

Proof. An ideal $A$ of $R$ is called right $t$-nilpotent if for any sequence $\left(a_{1}, a_{2}, a_{3}, \ldots\right)$ of elements of $A$ the product $a_{n} \ldots a_{2} a_{1}=0$ for some $n$. An unpublished lemma of G. Azumaya shows that an ideal $A$ is right $t$-nilpotent if and only if $\operatorname{Ann}_{M}(A) \neq 0$ for all modules $R^{M}$, and so it follows from Corollary 2.2 that $\mathcal{L F}\left(H^{P}\right)$ contains only the zero module if and only if $\operatorname{tr}_{R}(P)$ is right $t$-nilpotent.

Proposition (2.4) Let $P_{R}$ be a right $R$-module, and let $T:{ }_{R} M \rightarrow z^{M}$ be the functor $T=P \Theta_{R}$. For $M \epsilon_{R} \mathbb{M}, M_{R}^{*}=\operatorname{Hom}_{Z}(M, Q / Z)_{R}$ will denote the character module of $M$.

(a) $M \in \mathfrak{L F}(T)$ if $P_{R}$ generates $M_{R}^{*}$.

(b) If $P_{R}$ is flat, then $M \in \mathfrak{L F}(T) \Leftrightarrow \operatorname{Hom}_{R}\left(P, M_{0}^{*}\right) \neq 0$ for all nonzero $R$-submodules $M_{0} \subseteq M$.

(c) If $P_{R}$ is projective, then $\mathfrak{W F}(T)=\mathscr{L F}\left(H^{A}\right)$, for $A=\operatorname{tr}_{R}\left(P_{R}\right)$.

Proof. (a) The natural isomorphism $\operatorname{Hom}_{Z}\left(P \otimes_{R} M, Q / Z\right) \simeq$ $\operatorname{Hom}_{R}(P, \operatorname{Hom}(M, Q / Z))$ shows that the following diagram is commutative.

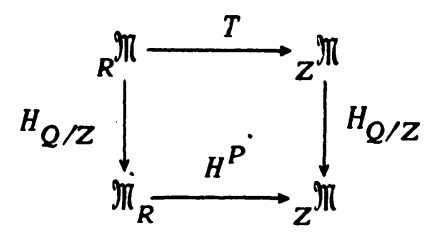

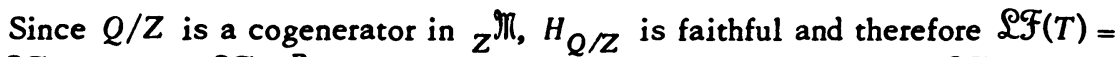
$\mathfrak{L F}\left(H_{Q / Z} T\right)=\mathfrak{L F}\left(H^{P} H_{Q / Z}\right)$. Since $H_{Q / Z}$ is contravariant, $M \in \mathfrak{L F}(T)$ if $M_{R}^{*}=H_{Q / Z}(M) \in R \mathcal{F}\left(H^{P}\right)$.

(b) Since $T$ is right exact, the dual of [2, Proposition 2.3] shows that $M \in \mathscr{L F}(T)$ if and only if $\operatorname{Hom}_{R}\left(P, i^{*}\right): \operatorname{Hom}_{R}\left(P, M^{*}\right) \rightarrow \operatorname{Hom}_{R}\left(P, M_{0}^{*}\right)$ is nonzero for the homomorphism $i^{*}$ induced by the inclusion mapping $i: M_{0} \rightarrow M$ for any nonzero submodule $M_{0}$ of $M$. When $T$ is exact, this can be strengthened to condition (b), again by using the dual of [2, Proposition 2.3].

(c) If $P_{R}$ is projective, then $P \otimes_{R} M=0$ if and only if $A M=0$, where $A=\operatorname{tr}_{R}\left(P_{R}\right)$. Since $T$ is exact, $M \in \mathfrak{L F}(T)$ if and only if $M$ has no nonzero submodules in $\operatorname{Ker}(T)$, and this occurs if and only if $\operatorname{Ann}_{M}(A)=0$. Because $A$ is an idempotent ideal, it is a trace ideal when viewed as a left ideal, and applying Corollary 2.2 shows that $\mathfrak{L F}(T)=\mathscr{L F}\left(H^{A}\right)$. 
A class of modules is called a $T T F$-class if it is both a torsion class and a torsionfree class. There is a one-to-one correspondence between TTF-classes and idempotent ideals of the ring $R$, and this correspondence is given by associating to an idempotent ideal $A$ the class of modules annihilated by $A$ [6]. The corresponding torsionfree class is then just the class of modules $M$ such that $\operatorname{Ann}_{M}(A)=0$. This proves the following proposition.

Proposition (2.5) A class $\mathcal{C}$ of modules is the torsionfree class of a TTF. class $\Leftrightarrow \mathcal{C}=\mathfrak{L F}\left(H^{A}\right)$ for an idempotent ideal $A$.

Proposition (2.6) For a module ${ }_{R} P$ such that $P \in \mathfrak{L F}\left(H^{P}\right), \mathfrak{L F}\left(H^{P}\right)$ is a $T T F$-class $\Leftrightarrow \mathfrak{L F}\left(H^{P}\right)=\mathfrak{L F}\left(H^{A}\right)$ for an ideal $A=$ Re generated by a central idempotent $e$.

Proof. Let $\mathcal{C}$ be the torsion class corresponding to $\mathcal{Q F}\left(H^{P}\right)$, and let $\mathscr{D}=\left\{M: \operatorname{Hom}_{R}(X, M)=0\right\}$ for all $X \in \mathcal{Q F}\left(H^{P}\right)$.

$(\Rightarrow)$. Since $H^{P}$ is left exact, Theorem 1.8 shows that $\mathcal{C}$ is hereditary. Furthermore, $\mathfrak{D}$ is closed under factor modules, since if $M \in \mathfrak{D}, 0 \neq p:{ }_{R} M \rightarrow{ }_{R} N$ is an epimorphism and $0 \neq f: X \rightarrow N$, for $X \in \mathfrak{L F}\left(H^{P}\right)$, then since $\mathscr{L F}\left(H^{P}\right)$ is a $T T F$-class it is closed under epimorphisms and so $f(X) \in \mathfrak{L F}\left(H^{P}\right)$. Thus for $M_{0}=p^{-1}(f(X)), 0 \neq p: M_{0} \rightarrow f(X)$, and hence there exists $g: P \rightarrow M_{0}$ with $p g \neq 0$. This is impossbile since $P \in \mathscr{Q F}\left(H^{P}\right)$ and $M_{0} \in \mathfrak{D}$. By [1, Corollary 7], $\mathcal{C}=\mathfrak{D}$ and $\mathcal{L F}\left(H^{P}\right)=\{M: B M=0\}$ for a principal left ideal $B$ generated by a central idempotent $e$. If $A=R(1-e)$, then $B M=0 \Leftrightarrow \mathrm{Ann}_{M}(A)=0$, so by Corollary 2.2, $\mathscr{E F}\left(H^{P}\right)=\mathscr{L F}\left(H^{A}\right)$.

$(\Leftarrow)$. If $A=R e$, then let $B=R(1-e)$. Then it follows from Corollary 2.2 that $\mathfrak{L F}\left(H^{A}\right)=\left\{M: A_{n}(A)=0\right\}=\{M: B M=0\}$. Since $B$ is an idempotent ideal, the correspondence between idempotent ideals and $T T F$-classes shows that $\mathfrak{Q F}\left(H^{A}\right)$ is a $T T F$-class.

Note that if $\mathscr{L F}\left(H^{P}\right)=\mathfrak{R F}\left(H^{P}\right)$, then $P \in \mathfrak{L F}\left(H^{P}\right)$ and $\mathscr{L F}\left(H^{P}\right)$ is a TTF-class. Thus if $R$ has no central idempotents other than 0 and 1 , then for

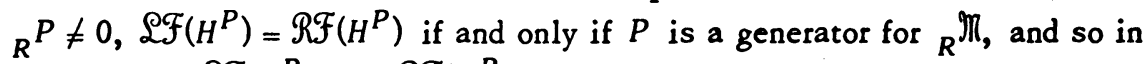
such a case $\mathscr{L F}\left(H^{P}\right)$ and $\mathscr{R F}\left(H^{P}\right)$ rarely coincide. On the other hand, if $A=R e$ for a central idempotent $e$, then $\mathcal{L F}\left(H^{A}\right)$ is just the full subcategory of $A$-modules, and so $\mathscr{L F}\left(H^{A}\right)=\mathscr{R F}\left(H^{A}\right)$.

Proposition (2.7) For $P \in \in_{R} \mathbb{M}, \mathscr{R F}\left(H^{P}\right)$ is closed under products if and only if $P$ is finitely generated as a module over $\operatorname{End}_{R}(P)$.

Proof. $(\Rightarrow)$. If $R \mathcal{F}\left(H^{P}\right)$ is closed under products, then $P$ generates $\Pi_{\alpha \in I} P_{\alpha}$, where $P_{\alpha}=P$ and the index set $I$ is $P$ itself. Let $x \in \Pi_{\alpha \in I} P_{a}$ be the element with $p$ th component $x_{p}=p$. Then $x=\sum_{j=1}^{n} f_{j}\left(p_{j}\right)$ for elements 
$p_{j} \in P$ and $f_{j} \in \operatorname{Hom}_{R}\left(P, \Pi_{\alpha \in I} P_{\alpha}\right)$. For the projections $\pi_{p}: \Pi_{\alpha \in I} P_{a} \rightarrow P_{p}$, $p=\pi_{p}(x)=\sum_{j=1}^{n} \pi_{p} f_{j}\left(p_{j}\right)$, with $\pi_{p} f_{j} \in$ End ${ }_{R}(P)$. Thus $P$ is generated over End $_{R}(P)$ by the elements $p_{1}, \cdots, p_{n}$.

$(\Leftarrow)$. If the modules $\left\{M_{\alpha}\right\}_{\alpha \in I}$ are each generated by $P$, then given $x \in \Pi_{\alpha \in I} M_{\alpha}$ the $\alpha$ th component satisfies $x_{\alpha}=\sum_{i=1}^{m} f_{i}^{\alpha}\left(x_{i}^{\alpha}\right)$ for elements $x_{i}^{\alpha} \in P$ and $f_{i}^{\alpha} \epsilon$ $\operatorname{Hom}_{R}\left(P, M_{a}\right)$. Since $P$ is generated over $\operatorname{End}_{R}(P)$ by finitely many elements, say $p_{1}, \cdots, p_{n}$, there exist elements $g_{i j} \in \operatorname{End}_{R}(P)$ with $x_{i}^{\alpha}=\sum_{j=1}^{n} g_{i j}\left(p_{j}\right)$. Let $b_{j}^{\alpha}=\sum_{i=1}^{m} f_{i}^{a} g_{i j}$. Then $x_{a}=\sum_{j=1}^{n} b_{j}^{\alpha}\left(p_{j}\right)$, and for $b_{j}: P \rightarrow \Pi_{\alpha \in I} M_{a}$ given by $b_{j}=\Pi b_{j}^{a}, x=\sum_{j=1}^{n} b_{j}\left(p_{j}\right)$, which shows that $\Pi_{a \in I} M_{a}$ is generated by $P$, and thus $\Pi_{a \in I} M_{a} \in \mathscr{R F}\left(H^{P}\right)$.

The above proposition is related to Proposition 3.6 of [4]. The proof of the next proposition is easy, so it has been omitted. A similar result is that $\mathscr{R F}\left(H^{P}\right)$ $=\mathscr{L F}\left(H_{P}\right)$ if and only if $P$ is a generator and a cogenerator as an $R / A n n(P)$-module.

Proposition (2.8) The following conditions are equivalent for $P \in \epsilon_{R}$ M.

(a) $\mathfrak{R F}\left(H^{P}\right)$ is closed under submodules and products.

(b) $P$ is a generator as an $R / A \mathrm{nn}(P)$-module.

(c) $R \mathcal{F}\left(H^{P}\right)$ is the full subcategory of $R / A \mathrm{nn}(P)$-modules.

3. T-faithful Giraud subcategories. A Giraud subcategory of $R^{\mathbb{M}}$ is a full reflective subcategory whose reflector is exact. (See [13].) Such a subcategory is a Grothendieck category, and in fact any Grothendieck category is equivalent to a Giraud subcategory of some category of modules. Throughout this section $T:{ }_{R} \mathbb{R} \rightarrow \mathfrak{B}$ will be a fixed additive functor into an abelian category $\mathfrak{B}$.

Definition (3.1) The full subcategory of $R^{M}$ determined by all modules ${ }_{R} M$ such that there exists an injective resolution $0 \rightarrow M \rightarrow X_{0} \rightarrow X_{1} \rightarrow \ldots$ with $X_{0} \in \mathfrak{L F}(T)$ will be denoted by $\mathfrak{L F}_{1}(T)$. Similarly, $\mathfrak{L F}_{2}(T)$ will denote the full subcategory determined by all modules $R^{M}$ such that there exists an injective resolution $0 \rightarrow M \rightarrow X_{0} \rightarrow X_{1} \rightarrow X_{2} \rightarrow \ldots$ with $X_{0}$ and $X_{1}$ in $\mathfrak{L F}(T)$.

If $T$ is a monofunctor, then $\mathscr{L F}(T)$ is closed under essential extensions, so $\mathscr{L F}(T)=\mathfrak{L F}_{1}(T)$. This is not the case in general, since for the functor $H_{Z}:{ }_{Z} \mathbb{M} \rightarrow$ $z$, $\mathfrak{Q F}\left(H_{Z}\right)$ is the class of torsionless abelian groups, but $\mathfrak{L F}_{1}\left(H_{Z}\right)$ contains only the zero group since no nonzero divisible group is torsionless. If ${ }_{R} V$ is an injective module, then $M \in \mathfrak{L F}_{2}\left(H_{V}\right)$ if and only if $V$-dom $\operatorname{dim} M \geq 2$, as is shown by Storrer [7, Proposition A.5]. The definition is thus motivated by the notion of dominant dimension used by Tachikawa in [15].

Lemma (3.2) Let $M \epsilon_{R}$ M, with injective envelope $E(M)$.

(a) $M \in \mathfrak{L F}_{1}(T) \Leftrightarrow E(M) \in \mathfrak{L F}(T)$.

(b) $M \in \mathcal{L F}_{2}(T) \Leftrightarrow E(M)$ and $E(E(M) / M)$ belong to $\mathcal{L F}(T)$. 
Proof. Each term in the minimal injective resolution of $M$, given by $0 \rightarrow M$ $\rightarrow E(M) \rightarrow E(E(M) / M) \rightarrow \ldots$, is a direct summand of the corresponding term in every injective resolution [7, Lemma A.3]. The result follows since $\mathfrak{L F}(T)$ is closed under submodules.

Proposition (3.3) $\mathfrak{S F}_{1}(T)$ is the torsionfree class of a torsion radical.

Proof. By Lemma $3.2(a), \mathcal{L F}_{1}(T)$ is closed under submodules, direct products, and injective envelopes. If $0 \rightarrow M^{\prime} \rightarrow M \rightarrow M^{\prime \prime} \rightarrow 0$ is an exact sequence in $\mathbb{R}^{M}$ with $M^{\prime}, M^{\prime \prime} \in \mathcal{L F}_{1}(T)$, then $M$ can be embedded in $E\left(M^{\prime}\right) \oplus E\left(M^{\prime \prime}\right)$, and so $M \in \mathcal{L F}_{1}(T)$.

Theorem (3.4) $\mathfrak{L F}_{2}(T)$ is a Giraud subcategory.

Proof. Since $\mathfrak{L F}_{1}(T)$ is the torsionfree class of a torsion radical, by [13, Theorem 10.2] there is a corresponding Giraud subcategory consisting of those modules ${ }_{R} M$ such that $E(M)$ and $E(E(M) / M)$ belong to $\mathcal{L F}_{1}(T)$. By Lemma 3.2 this is precisely $\mathfrak{L F}_{2}(T)$.

By construction, $\mathscr{L F}_{1}(T)$ is the largest torsionfree class (of a torsion radical) which is contained in $\mathscr{E F}(T)$, and similarly, $\mathscr{L F}_{2}(T)$ is the largest Giraud subcategory contained in $\mathscr{L F}(T)$, so $\mathfrak{L F}_{2}(T)$ can be characterized as the largest left $T$-faithful Giraud subcategory of $R^{\Re}$. Theorem 3.4 can also be deduced from [9, Chapter V, Theorem 6.8] and the following proposition, for which it is interesting to have a proof which uses only Lemma 3.2.

Proposition (3.5) Let $0 \rightarrow M^{\prime} \rightarrow M \rightarrow M^{\prime \prime} \rightarrow 0$ be an exact sequence in ${ }_{R} M$, with $M \in \mathfrak{L F}_{1}(T)$. If $M^{\prime} \in \mathfrak{S F}_{2}(T)$, then $M^{\prime \prime} \in \mathfrak{L F}_{1}(T)$ and the converse bolds if $M \in \mathfrak{L F}_{2}(T)$.

Proof. Assume that $M \in \mathscr{L F}_{2}(T)$. Then $E(M)=E\left(M^{\prime}\right) \oplus X$, where ${ }_{R} X$ is injective, and $X \in \mathscr{L F}(T)$ since $M \in \mathfrak{L F}_{1}(T)$. Now $M^{\prime \prime} \simeq M / M^{\prime} \subseteq E(M) / M^{\prime}=$ $\left(E\left(M^{\prime}\right) / M^{\prime}\right) \oplus X$, and so $E\left(M^{\prime \prime}\right) \simeq E\left(M / M^{\prime}\right) \subseteq E\left(E\left(M^{\prime}\right) / M^{\prime}\right) \oplus X$. The last module belongs to $\mathscr{L F}(T)$ by Lemma 3.2 since $M^{\prime} \in \mathscr{L F}_{2}(T)$, so $E\left(M^{\prime \prime}\right) \in \mathfrak{L F}(T)$ and thus $M^{\prime \prime} \in \mathscr{L F}_{1}(T)$.

Conversely, assume that $M \in \mathfrak{L F}_{2}(T)$ and $M^{\prime \prime} \in \mathfrak{L F}_{1}(T)$. First, $E\left(M^{\prime}\right) \subseteq$ $E(M) \epsilon \mathfrak{L F}(T)$. Next, let $f: E(M) \rightarrow(E(M) / M) \oplus E\left(M^{\prime \prime}\right)$ be the homomorphism induced by the projections $E(M) \rightarrow E(M) / M$ and $M \rightarrow M^{\prime \prime}$. Then ker $(f)=M^{\prime}$ since $f(m)=0$ implies $m \in M$ and therefore $m \in M^{\prime}$, by examining the first component of the homomorphism and then the second. Thus $E\left(M^{\prime}\right) / M^{\prime} \subseteq E(M) / M^{\prime}$ is isomorphic to a submodule of $E(E(M) / M) \oplus E\left(M^{\prime \prime}\right)$, which belongs to $\mathscr{L F}(T)$ by assumption, and so it follows that $E\left(E\left(M^{\prime}\right) / M^{\prime}\right) \in \mathscr{L F}(T)$. By Lemma 3.2 this completes the proof. $\square$ 
Proposition (3.6) If $T$ is exact and $\operatorname{Ker}(T)$ is an bereditary torsion class,

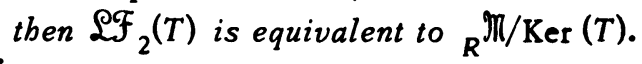

Proof. If $T$ is exact and $\operatorname{Ker}(T)$ is an hereditary torsion class, then by Theorem 1.8, $\mathscr{E F}(T)$ is the torsionfree class determined by $\operatorname{Ker}(T)$ and $\mathfrak{L F}_{2}(T)$ is the Giraud subcategory corresponding to $\operatorname{Ker}(T)$. It then follows from [13, Corollary 7.9] that $\mathfrak{L F}_{2}(T)$ is equivalent to the quotient category ${ }_{R} \mathbb{M} / \operatorname{Ker}(T)$ constructed in the usual way. (See [13] or [14].)

Theorem (3.7) Let $\mathbb{Q}$ be a Giraud subcategory of $R^{\text {MI }}$ with exact reflector $Q:{ }_{R} M \rightarrow Q$. The following conditions are equivalent:

(a) $\mathfrak{Q}$ is a subcategory of $\mathfrak{Q F}_{2}(T)$.

(b) $Q$ is $T$-faitbful and $T(f)=0$ implies $T Q(f)=0$, for all $R$-bomomorphisms $f$.

(c) $T(f)=0$ implies $Q(f)=0$, for all R-bomomorphisms $f$.

(d) If $M^{\prime} \rightarrow M \rightarrow M^{\prime \prime}$ is any sequence of $R$-modules, then if the image under $T$ of $M^{\prime} \rightarrow M \rightarrow M^{\prime \prime}$ is exact, so is $Q\left(M^{\prime}\right) \rightarrow Q(M) \rightarrow Q\left(M^{\prime \prime}\right)$.

Proof. Since $\mathfrak{L F}_{2}(T) \subseteq \mathfrak{L F}(T)$, (a) $\Rightarrow$ (b) and $(b) \Rightarrow$ (c) follow directly from Theorem 1.4. Assume that $T$ is covariant.

(c) $\Rightarrow$ (d). Suppose that $M^{\prime} \stackrel{f}{\rightarrow} M \stackrel{g}{\rightarrow} M^{\prime \prime}$ is a sequence such that $T\left(M^{\prime}\right) \stackrel{T(f)}{\longrightarrow}$ $T(M) \stackrel{T(g)}{\longrightarrow} T\left(M^{\prime \prime}\right)$ is exact in $B$. Then $T(g f)=0$ implies $Q(g f)=0$. Let $u: K \rightarrow M$ be the kernel of $g$ and $p: M \rightarrow C$ be the cokernel of $f$. Then $T(u)$ factors through the kernel of $T(g)$ and $T(p)$ factors through the cokernel of $T(g)$. Hence $T(p u)=0$, and this implies that $Q(p u)=0$. But $Q$ is exact, so it preserves kernels and cokernels, which means that $Q(u)=\operatorname{ker}(Q(g))$ and $Q(p)=$ coker $(Q(f))$. Since $Q(p) Q(u)=0$, this implies that $Q\left(M^{\prime}\right) \rightarrow Q(M) \rightarrow Q\left(M^{\prime \prime}\right)$ is exact.

(d) $\Rightarrow$ (c). Suppose that $T(f)=0$ for $f: M \rightarrow M^{\prime \prime}$. Then for the identity $1: M \rightarrow M$, the sequence $T(M) \stackrel{1}{\rightarrow} T(M) \stackrel{T(\cap)}{\longrightarrow} T\left(M^{\prime \prime}\right)$ is exact. By assumption, $Q(M) \stackrel{1}{\rightarrow} Q(M) \stackrel{Q(\cap)}{\longrightarrow} Q\left(M^{\prime \prime}\right)$ is exact, which implies $Q(f)=0$.

(c) $\Rightarrow$ (a). If $T(f)=0$ implies $Q(f)=0$, then by Theorem 1.4, $\mathfrak{Q} \subseteq \mathfrak{L F}(T)$. If $A \in \mathbb{Q}$, then since $\mathfrak{Q}$ is abelian and has enough injectives, there exists an injective resolution $0 \rightarrow A \rightarrow X_{0} \rightarrow X_{1}$ in $\mathcal{Q}$. The inclusion functor is left exact

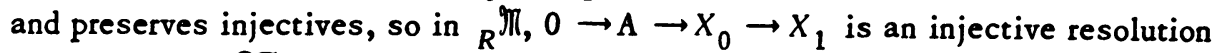
with $X_{0}, X_{1} \in \mathfrak{L F}(T)$. By definition, $A \in \mathfrak{L F}_{2}(T)$.

Lemma (3.8) If $T=T Q$ for the reflector $Q:{ }_{R} \pi \rightarrow \mathcal{L F}_{2}(T)$ and $Q$ is a $T$.

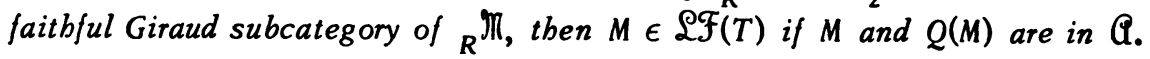

Proof. If $M, Q(M) \in \mathfrak{Q}$, let $\phi_{M}: M \rightarrow Q(M)$ be the homomorphism given by the adjoint situation. Since $\mathbb{Q}$ is a Giraud subcategory, it follows that $\operatorname{ker}\left(\phi_{M}\right) \in \mathbb{Q}$. For the identity 1 of $\operatorname{ker}\left(\phi_{M}\right)$ it follows that $T(1)=T Q(1)=0$, and then since $\mathbb{Q}$ 
is $T$-faithful, $1=0$ and $\operatorname{ker}\left(\phi_{M}\right)=0$, which shows that $M \in \mathfrak{L F}(T)$.

Note that it is not true in general that $T=T Q$, since it is possible to have $T \neq 0$ but $Q=0$, for example $T=H_{z}: z^{M} \rightarrow z^{M}$. Recall that an hereditary torsion class is called stable if it is closed under essential extensions.

Proposition (3.9) If $T$ is exact and $\operatorname{Ker}(T)$ is a stable, bereditary torsion class, then $\mathfrak{L F}_{2}(T)$ is the largest $T$-faithful Giraud subcategory of ${ }_{R} \mathbb{M}$.

Proof. To show that $\mathfrak{L F}_{2}(T)$ is a maximal $T$-faithful Giraud subcategory it is not necessary to assume that $\operatorname{Ker}(T)$ is stable. If $T$ is exact and $\operatorname{Ker}(T)$ is an hereditary torsion class, then $T=T Q$ by $[14, \mathrm{p} .40]$, so if $\mathcal{Q}$ is a Giraud subcategory containing $\mathcal{L F}_{2}(T)$ which is $T$-faithful, then the conditions of Lemma 3.8 are satisfied for each $M \in \mathbb{Q}$, and so $\mathfrak{Q} \subseteq \mathfrak{Q F}(T)$. Since $\mathfrak{L F}_{2}(T)$ is the largest Giraud subcategory contained in $\mathscr{L F}(T), \mathfrak{Q} \subseteq \mathfrak{L F}_{2}(T)$.

If $Q^{\prime}:{ }_{R} \mathbb{M} \rightarrow Q$ is the reflection into a $T$-faithful Giraud subcategory, and $M \in \operatorname{Ker}(T)$, let $M \rightarrow M^{\prime \prime} \rightarrow Q^{\prime}(M)$ be the factorization of the natural homomorphism $M \rightarrow Q^{\prime}(M)$ into an epimorphism followed by a monomorphism. Then $M^{\prime \prime} \in \operatorname{Ker}(T)$ since $M \rightarrow M^{\prime \prime}$ is an epimorphism, and since $\operatorname{Ker}(T)$ is stable, $Q^{\prime}(M) \in \operatorname{Ker}(T)$ because $M^{\prime \prime} \rightarrow Q^{\prime}(M)$ is an essential monomorphism. Thus $T Q^{\prime}(M)=0$ and so $Q^{\prime}(M)=0$ since $\mathcal{Q}$ is $T$-faithful. Finally, $\mathcal{Q} \subseteq \mathscr{F F}_{2}(T)$ since $\operatorname{Ker}\left(Q^{\prime}\right) \supseteq \operatorname{Ker}(T)=\operatorname{Ker}(Q)$.

Corollary (3.10) If $R$ is commutative and Noetherian, and $T$ is covariant and bas a right adjoint, then $\mathfrak{L F}_{2}(T)$ is characterized as the largest $T$-faitbful Giraud subcategory of ${ }_{R}$ M.

Proof. Since $T$ has an adjoint, $\operatorname{Ker}(T)$ is an hereditary torsion class. Furthermore, $\operatorname{Ker}(T)$ is stable since $R$ is commutative and Noetherian [13, Proposition 5.12].

\section{Applications.}

Proposition (4.1) Let $\mathfrak{B}$ be an additive category, and suppose the covariant functor $S:{ }_{R} \Re \rightarrow B$ has a left adjoint $T$, with $\psi: T S \rightarrow$ Id the associated natural transformation. Let $A=\operatorname{Im}\left(\psi_{R}\right)$.

(a) ${ }_{R} M \in \mathscr{L F}(S) \Rightarrow \operatorname{Ann}_{M}(A)=0$.

(b) ${ }_{R}^{M} \in \mathscr{L F}_{2}(S) \Leftrightarrow$ the natural mapping $R_{R}^{M} \rightarrow{ }_{R} \operatorname{Hom}_{R}(A, M)$ is an isomorphism.

Proof. (a) For any homomorphism $f: R \rightarrow M$, the following diagram is commutative. 


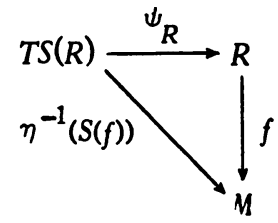

Thus $S(f) \neq 0 \Leftrightarrow \eta^{-1}(S(f)) \neq 0 \Leftrightarrow f \psi_{R} \neq 0$, and so $S(f) \neq 0 \Leftrightarrow f(A) \neq 0$. It follows from this that if $M \in \mathscr{L F}(S)$, then $\operatorname{Hom}_{R}(R / A, M)=0$. Conversely, if $f:{ }_{R} X \rightarrow{ }_{R} M$, then there exists $g: R \rightarrow X$ with $f g \neq 0$, and if $\operatorname{Hom}_{R}(R / A, M)=0$, then $f g(A) \neq 0$, which implies $S(f g) \neq 0$ and therefore $S(f) \neq 0$, so that $M \in \mathfrak{L F}(S)$. This proves (a), since $\operatorname{Ann}_{M}(A)=0 \Leftrightarrow \operatorname{Hom}_{R}(R / A, M)=0$.

(b) This follows immediately from (a) and the characterization of the quotient category determined by an ideal $A$ given by Mueller [12, Proposition 1]. Note that $S$ is a monofunctor, and so $\mathfrak{P F}_{1}(S)=\mathscr{L F}(S)$.

Proposition (4.2) In the above situation, the inclusion functor from $\mathfrak{L F}_{2}(S)$ into ${ }_{R}$ M preserves direct sums.

Proof. If $\left\{M_{a}\right\}_{a \in I}$ is a set of modules in $\mathfrak{S F}_{2}(S)$, then the factor module $E\left(\bigoplus_{\alpha \in I} M_{\alpha}\right) / \bigoplus_{\alpha \in I} M_{\alpha}$ is in $\mathcal{F F}(S)$. To show this, if $x \in E\left(\bigoplus_{\alpha \in I} M_{\alpha}\right)$, with $A x \in \bigoplus_{a \in I} M_{a}$, then $A x \in \bigoplus_{a \in F} M_{\alpha}$ for some finite subset $F$ of $I$. But this finite direct sum is in $\mathfrak{L F}_{2}(S)$, and so $E\left(\bigoplus_{\alpha \in I} M_{\alpha}\right) / \bigoplus_{\alpha \in F} M_{\alpha}$ belongs to $\mathfrak{L F}(S)$, and therefore $x \in \bigoplus_{\alpha \in F} M_{\alpha}$. Thus by Proposition 3.5, $\bigoplus_{\alpha \in I} M_{\alpha}$ belongs to $\mathcal{L F}_{2}(S)$.

Note that this proof applies to the quotient category determined by any ideal, and so in particular it applies to $\mathcal{L F}_{2}(T)$ for $T=P \otimes_{R}$, where $P_{R}$ is projective.

A Morita context consists of two rings $R$ and $R^{\prime}$ and two bimodules ${ }_{R^{\prime}} P_{R}$ and ${ }_{R}{ }^{M} R^{\prime}$ together with two bimodule homomorphisms $(-,-):{ }_{R}{ }^{M} \otimes_{R^{\prime}} P_{R} \rightarrow R$ and $[-,-]:{ }_{R^{\prime}} P \otimes_{R^{\prime}} M_{R^{\prime}} \rightarrow R^{\prime}$ satisfying the conditions $m\left[p, m^{\prime}\right]=(m, p) m^{\prime}$ and $p\left(m, p^{\prime}\right)=[p, m] p^{\prime}$ for all $p, p^{\prime} \in P$ and $m, m^{\prime} \in M$. Let $A$ and $A^{\prime}$ be the respective images of the maps $(-,-)$ and $[-,-]$. A context is nondegeneratable if the two ideals $A$ and $A^{\prime}$ are faithful as left and right modules [12, Proposition

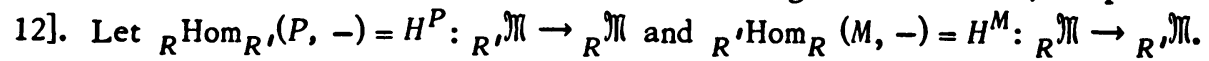

Theorem (4.3) Let $\langle P, M\rangle$ be a nondegeneratable Morita context. Then the categories $\mathfrak{L F}_{2}\left(H^{P} H^{M}\right)$ and $\mathfrak{L F}_{2}\left(H^{M} H^{P}\right)$ are equivalent.

Proof. With the notation previously given, the result holds if it is merely assumed that $A_{R}$ and ${ }_{R^{\prime}} A^{\prime}$ are faithful. By [12, Theorem 3], if $A_{R}$ is faithful, then $\mathrm{Ann}_{R} A=0$ and so every $R$-homomorphism $f:{ }_{R} M \otimes_{R^{\prime}} P \rightarrow{ }_{R} R$ factors through $A$, so that $A$ is the trace ideal of the module ${ }_{R} M \otimes_{R^{\prime}} P$. Similarly, 
$A^{\prime}$ is the trace ideal of $P \otimes{ }_{R} M_{R^{\prime}}$. Using Corollary 2.2 to translate Mueller's terminology into that of this paper, [12, Theorem 3] shows that the categories $\mathfrak{L F}_{2}\left(H^{A}\right)$ and $\mathfrak{L F}_{2}\left(H^{A^{\prime}}\right)$ are equivalent. By Corollary 2.2 and the fact that $A$ is the trace ideal of ${ }_{R} M \otimes_{R^{\prime}} P$, it follows that $\mathscr{P F}_{2}\left(H^{M \otimes P}\right)$ is equivalent to $\mathscr{L F}_{2}\left(H^{P \otimes M}\right)$. This completes the proof, since $\operatorname{Hom}_{R}\left(M \otimes_{R^{\prime}} P,-\right)$ is naturally equivalent to $\operatorname{Hom}_{R^{\prime}}\left(P, \operatorname{Hom}_{R}(M,-)\right)$ and $\operatorname{Hom}_{R^{\prime}}\left(P \otimes_{R} M,-\right)$ is naturally equivalent to $\operatorname{Hom}_{R}\left(M, \operatorname{Hom}_{R^{\prime}}(P,-)\right)$.

Until noted otherwise, $T:{ }_{R} \mathbb{M} \rightarrow \mathfrak{B}$ will be a covariant functor, with right adjoint $S$. If $T$ is exact, then $\operatorname{Ker}(T)$ is an hereditary torsion class, and $\mathfrak{L F}_{2}(T)$ is the corresponding Giraud subcategory. For any $B \in \mathbb{B}$ and any exact sequence $0 \rightarrow M^{\prime} \rightarrow M \rightarrow M^{\prime \prime} \rightarrow 0$ in $R^{M}$ with $M^{\prime \prime} \in \operatorname{Ker}(T)$, the natural isomorphism

$$
\begin{gathered}
0 \rightarrow \operatorname{Hom}_{R}\left(M^{\prime \prime}, S(B)\right) \rightarrow \operatorname{Hom}_{R}(M, S(B)) \rightarrow \operatorname{Hom}_{R}\left(M^{\prime}, S(B)\right) \\
\downarrow \\
\downarrow \rightarrow \operatorname{Hom}_{\mathcal{B}}\left(T\left(M^{\prime \prime}\right), B\right) \rightarrow \operatorname{Hom}_{\mathcal{B}}(T(M), B) \rightarrow \operatorname{Hom}_{\mathcal{B}}\left(T\left(M^{\prime}\right), B\right)
\end{gathered}
$$

shows, since $T\left(M^{\prime \prime}\right)=0$, that $S(B)$ is torsionfree and injective with respect to $\operatorname{Ker}(T)$. Hence $S(B) \in \mathfrak{L F}_{2}(T)$. The module of quotients for any $M \epsilon_{R} \mathbb{M}$ is then given by $\operatorname{ker}\left(\phi_{S T(M)}-S T\left(\phi_{M}\right)\right)$, as is shown by Proposition 4.5. Using $\operatorname{rad}_{T}$ this can be generalized to functors which are not necessarily exact.

Definition (4.4) Let $T:{ }_{R} \mathbb{M} \rightarrow \mathfrak{B}$ be covariant, with right adjoint $S$. For any $M \epsilon_{R} M$, define $Q_{T}(M)=\operatorname{ker}\left(\phi_{S T(M)}-S T\left(\phi_{M}\right)\right)$, where $\phi_{M}: M \rightarrow S T(M)$ and $\phi_{S T(M)}: S T(M) \rightarrow S T S T(M)$ are the homomorphisms determined by the adjoint situation.

Proposition (4.5) Let $T:{ }_{R} \Uparrow \rightarrow \mathfrak{B}$ bave a right adjoint $S$.

(a) For any $B \in \mathfrak{B}, Q_{T}(S(B))=S(B)$.

(b) For any $M \in \epsilon_{R}$ M, $Q_{T}(M)$ is the $\operatorname{rad}_{T}$-closure of $\phi_{M}(M)$ in $S T(M)$.

(c) For any $M \in \epsilon_{R} \mathbb{M}, Q_{T}(M) \in \mathfrak{L F}(T)$, and $Q_{T}(M)$ is a direct summand of $Q_{T}\left(Q_{T}(M)\right)$.

Proof. Recall that for a radical $\rho$, the $\rho$-closure of a submodule $M^{\prime}$ in $M$ is the inverse image in $M$ of $\rho\left(M / M^{\prime}\right)$. Equivalently, it is the intersection of all submodules of $M$ which contain $M^{\prime}$ and have $\rho$-torsionfree quotient.

(a) $S(B)$ is a direct summand of $S T S(B)$, since $S\left(\psi_{B}\right) \phi_{S(B)}=1_{S(B)}$, and so $S T S(B) / \phi_{S(B)}(S(B))$ is $\operatorname{rad}_{T}$ torsionfree. Part (b) shows that $Q_{T}(S(B))=S(B)$.

(b) The diagram 


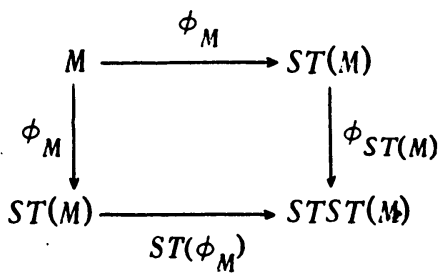

is commutative, and so $\left(\phi_{S T(M)}-S T\left(\phi_{M}\right)\right) \phi_{M}=0$. Thus $\phi_{M}(M) \subseteq Q_{T}(M)$.

It follows immediately from the definition of $Q_{T}(M)$ that it is $\operatorname{rad}_{T}$-closed in $S T(M)$, since $S T S T(M) \in \mathscr{L F}(T)$. Thus $Q_{T}(M)$ contains the $\operatorname{rad}_{T}$-closure of $\phi_{M}(M)$. On the other hand, if $f: S T(M) \rightarrow N$, with $N \in \mathscr{L} \mathcal{F}(T)$, then the following diagram is commutative

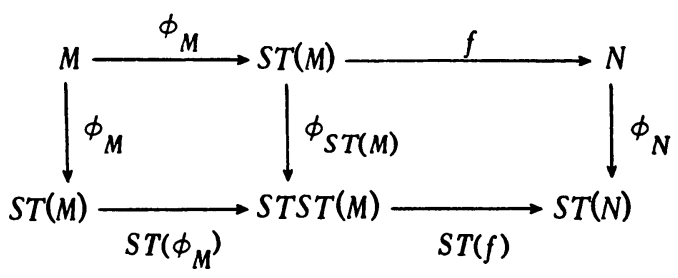

If $f \phi_{M}=0$, then $S T(f) S T\left(\phi_{M}\right)=0$ and so $S T(f) S T\left(\phi_{M}\right)\left(Q_{T}(M)\right)=0$. But then $S T(f) \phi_{S T(M)}\left(Q_{T}(M)\right)=0$ since $S T\left(\phi_{M}\right)=\phi_{S T(M)}$ when restricted to $Q_{T}(M)$. Thus $\phi_{N} f\left(Q_{T}(M)\right)=0$, which shows that $f\left(Q_{T}(M)\right)=0$, because $N \in \mathscr{L} \mathcal{F}(T)$ and consequently $\phi_{N}$ is a monomorphism. It now follows that $Q_{T}(M)$ is contained in the $\operatorname{rad}_{T}$-closure of $\phi_{M}(M)$.

(c) $Q_{T}(M) \in \mathscr{Q F}(T)$ since $S T(M) \in \mathscr{\varrho F}(T)$. The exact sequence

$$
0 \rightarrow Q_{T}(M) \rightarrow S T(M) \stackrel{\phi_{S T(M)}-S T\left(\phi_{M}\right)}{\longrightarrow} S T S T(M)
$$

shows, by the following lemma, that $Q_{T}(M)$ is a direct summand of $Q_{T}\left(Q_{T}(M)\right)$.

Lemma (4.6) Let $T:{ }_{R} \mathbb{M} \rightarrow \Re$, with right adjoint $S$, and let $M \in{ }_{R}$ M. The bomomorphism $\phi_{M}: M \rightarrow Q_{T}(M)$ splits if there exist $B \in \mathfrak{B}$ and $N \in \mathfrak{L} \mathcal{F}(T)$ together with an exact sequence $0 \rightarrow M \rightarrow S(B) \rightarrow N$.

Proof. Consider the following diagram, if the hypotheses are satisfied.

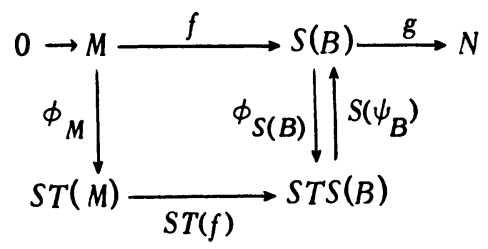

Then $g S\left(\psi_{B}\right) S T(f) \phi_{M}=g S\left(\psi_{B}\right) \phi_{S(B)} f=g f=0$ since $S\left(\psi_{B}\right) \phi_{S(B)}=11_{S(B)}$. Since 
$N \in \mathfrak{L F}(T)$, and $Q_{T}(M)$ is the $\operatorname{rad}_{T}$-closure of $M$ in $S T(M), g S\left(\psi_{B}\right) S T(f)\left(Q_{T}(M)\right)$ $=0$. Thus $S\left(\psi_{B}\right) S T(f)\left(Q_{T}(M)\right) \subseteq f(M)$, and so $f^{-1} S\left(\psi_{B}\right) S T(f)$ gives the splitting homomorphism, since $f$ is a monomorphism and $S\left(\psi_{B}\right) S T(f) \phi_{M}=f$.

In fact, the same proof can be used to show that $M \rightarrow Q_{T}(M)$ splits if there exist $B \in \mathfrak{B}, M^{\prime}, N \in \mathfrak{L F}(T)$, and an exact sequence $0 \rightarrow M \oplus M^{\prime} \rightarrow S(B) \rightarrow N$. This condition is also necessary.

Lemma (4.7) Let $T:{ }_{R} \mathbb{M} \rightarrow \mathscr{B}$, with right adjoint $S$. The following conditions are equivalent for any $M \epsilon_{R} \mathbb{M}$.

(a) $Q_{T}(M)=S T(M)$.

(b) The mapping $\operatorname{Hom}_{B}(T(M), T(N)) \rightarrow \operatorname{Hom}_{R}(S T(M), S T(N))$ induced by $S$ is onto for all $N \in \epsilon_{R}$ M.

(c) $\psi_{T(M)}$ is an isomorphism.

(d) $T\left(\phi_{M}\right)$ is an isomorphism.

(e) $\phi_{S T(M)}$ is an isomorphism.

Proof. Recall that for any $M \in{ }_{R} M, B \in \mathcal{B}, f: M \rightarrow S(B)$, and $g: T(M) \rightarrow B$, the adjoint mapping $\eta$ satisfies $\eta^{-1}(f)=\psi_{B} T(f)$ and $\eta(g)=S(g) \phi_{M}$. From the first it follows that $\psi_{T(M)} T\left(\phi_{M}\right)=1_{T(M)}$ and $S\left(\psi_{T(M)}\right) S T\left(\phi_{M}\right)=1_{S T(M)}$, and from the second that $S\left(\psi_{T(M)}\right) \phi_{S T(M)}=1 S T(M)$.

$(\mathrm{a}) \Rightarrow(\mathrm{b})$. Given $f: S T(M) \rightarrow S T(N)$, consider $g=\psi_{T(N)} T(f) T\left(\phi_{M}\right)$. Then

$$
\begin{aligned}
S(g) & =S\left(\psi_{T(N)}\right) S T(f) S T\left(\phi_{M}\right)=S\left(\psi_{T(N)}\right) S T(f) \phi_{S T(M)} \\
& =S\left(\psi_{T(N)}\right) \phi_{S T(N)} f=1_{S T(N)} f=f
\end{aligned}
$$

since $S T\left(\phi_{M}\right)=\phi_{S T(M)}$, and so (b) holds.

$(b) \Rightarrow(c)$. If (b) holds, then $\operatorname{Hom}_{B}(T(M), T S T(M)) \rightarrow \operatorname{Hom}_{R}(S T(M), \operatorname{STST}(M))$

is onto, so there exists $g \in \operatorname{Hom}_{\mathbb{B}}(T(M), T S T(M))$ with $S(g)=\phi_{S T(M)}$. Then $\eta\left(g \psi_{T(M)}\right)=S\left(g \psi_{T(M)}\right) \phi_{S T(M)}=S(g) S\left(\psi_{T(M)}\right) \phi_{S T(M)}=S(g)=\phi_{S T(M)}=\eta\left(1_{T(M)}\right)$, which implies $g \psi_{T(M)}=1_{T(M)}$, and so $\psi_{T(M)}$ is an isomorphism.

(c) $\Leftrightarrow$ (d). This is obvious, since $\psi_{T(M)} T\left(\phi_{M}\right)=1_{T(M)^{\circ}}$

(c) $\Rightarrow(e)$. If $\psi_{T(M)}$ is an isomorphism, then so is $S\left(\psi_{T(M)}\right)$, and so $\phi_{S T(M)}$ is an isomorphism since $S\left(\psi_{T(M)}\right) \phi_{S T(M)}=1_{S T(M)}$.

$(\mathrm{e}) \Rightarrow(\mathrm{a})$. If $\phi_{S T(M)}$ is an isomorphism, then so is $S\left(\psi_{T(M)}\right)$, and $S\left(\psi_{T(M)}\right) S T\left(\phi_{M}\right)=S\left(\psi_{T(M)}\right) \phi_{S T(M)}$ implies $S T\left(\phi_{M}\right)=\phi_{S T(M)} \cdot$

Note that since $\phi: S T \rightarrow S T S T$ is a natural transformation, a finite direct sum of modules satisfies the conditions of the lemma if and only if each summand satisfies the condition. If $f:{ }_{R} M \rightarrow{ }_{R} N$ and $S T(f)$ is an epimorphism, then condition (d) of Lemma 4.7 can be used to show that $Q_{T}(N)=S T(N)$ if $Q_{T}(M)=S T(M)$. Note that the last three conditions only require, respectively, that $\psi_{T(M)}$ is a 
monomorphism, that $T\left(\phi_{M}\right)$ is an epimorphism, or that $\phi_{S T(M)}$ is an epimorphism.

Proposition (4.8) Let $T:{ }_{R} \mathbb{M} \rightarrow \mathcal{B}$, with right adjoint $S$, and let $M, N \in{ }_{R} \mathbb{M}$ with $f: M \rightarrow N$ and $Q_{T}(N)=S T(N)$. Then $Q_{T}(M)=S T(M)$ if $T S T(f)$ is a monomorphism. This condition is satisfied if $T$ is exact and $\operatorname{ker}(f) \in \operatorname{Ker}(T)$.

Proof. Consider the following commutative diagram.

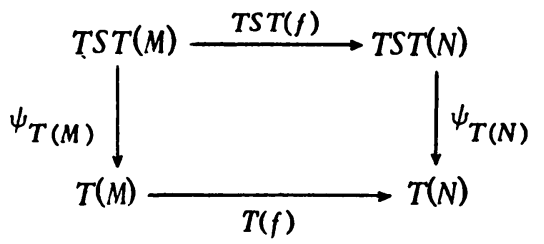

Since $Q_{T}(N)=S T(N), \psi_{T(N)}$ is a monomorphism, so $\psi_{T(N)} T S T(f)$ is also a monomorphism, and consequently $\psi_{T(M)}$ is a monomorphism. Thus $Q_{T}(M)=$ $S T(M)$ by Lemma 4.7.

If $T$ is exact, then $0 \rightarrow T(\operatorname{ker}(f)) \rightarrow T(M) \rightarrow T(N)$ is exact, and so $T(f)$ is a monomorphism. Since $T$ is exact and $S$ is a monofunctor, TST $(f)$ must be a monomorphism, and the previous condition holds.

Theorem (4.9) Let $T:{ }_{R} M \rightarrow B$, with right adjoint $S$, and let $M \epsilon_{R}{ }^{M}$. Then $\phi_{M}: M \rightarrow S T(M)$ is an isomorphism if and only if $Q_{T}(M)=S T(M)$ and there exists an exact sequence $0 \rightarrow M \rightarrow S(B) \rightarrow N$, for some $B \in \mathbb{B}$ and $N \in \mathfrak{L F}(T)$.

Proof. If $Q_{T}(M)=S T(M)$ and there exists an exact sequence $0 \rightarrow M \rightarrow S(B)$ $\rightarrow N$, with $B \in \mathscr{B}$ and $N \in \mathscr{L F}(T)$, then by Lemma $4.6 \phi_{M}: M \rightarrow Q_{T}(M)=S T(M)$ splits. By Proposition 4.5, $Q_{T}(M)$ is the $\operatorname{rad}_{T}$-closure of $\phi_{M}(M)$ in $S T(M)$, and if $\phi_{M}$ splits, then $\phi_{M}(M)$ is $\operatorname{rad}_{T}$-closed in $S T(M)$, so $\phi_{M}(M)=Q_{T}(M)=S T(M)$.

Conversely, if $\phi_{M}$ is an isomorphism, then the sequence $0 \rightarrow M \rightarrow S T(M)$ $\rightarrow 0$ is exact. $\square$

If $T$ is contravariant, then the above results can be dualized. In this case, a contravariant functor $S: \mathscr{B} \rightarrow{ }_{R} \mathbb{M}$ is said to be an adjoint on the right for $T$ if there exists a natural equivalence $\eta: \operatorname{Hom}_{\mathcal{B}}(\cdot, T(-)) \rightarrow \operatorname{Hom}_{R}(-, S(\cdot))$. For any $M \in{ }_{R} M$ and $B \in B, \eta$ induces $\phi_{M}=\eta\left(1_{T(M)}\right): M \rightarrow S T(M)$ and $\psi_{B}=\eta^{-1}\left(1_{S(B)}\right):$ $B \rightarrow T S(B)$.

The above results yield as corollaries several theorems of Morita [11]. If ${ }_{R} V \in{ }_{R} \mathbb{M}$, then $V$ is a right $E$-module for the opposite ring $E$ of $\operatorname{End}_{R}(V)$, and the functor $T=\operatorname{Hom}_{R}(-, V)_{E}:{ }_{R} \Uparrow \rightarrow \prod_{E}$ has an adjoint on the right $S=$ ${ }_{R} \operatorname{Hom}_{E}(-, V): \mathbb{M}_{E} \rightarrow{ }_{R}$ M. In this situation $\phi_{V}: V \rightarrow S T(V)$ is an isomorphism, and $S T(R)$ is the bicommutator End $_{E}(V)$ of $V$, which will be denoted by $\mathrm{Bic}_{R}(V)$. The module ${ }_{R} V$ is said to be of type $F_{b}$ if $\psi_{T(R)}$ is an isomorphism. (It follows from [10, Lemma 2.1] that this definition is equivalent to Morita's definition in 
[11].) If ${ }_{R} V$ is injective, then $Q_{T}(R)$ is the ring of quotients determined by $\operatorname{rad}_{T}$, as is shown by [7, Proposition 3.1]. It then follows from Lemma 4.7 that the ring of quotients determined by $V$ coincides with $\mathrm{Bic}_{R}(V)$ if and only if $V$ is of type $F_{b}$. Furthermore, since $V=Q_{T}(V)=S T(V)$, Proposition 4.8 implies the well-known result that the ring of quotients determined by $V$ coincides with $\operatorname{Bic}_{R}(V)$ if there exists a homomorphism $f:{ }_{R} R \rightarrow{ }_{R} V^{n}$ for some $n$, such that $\operatorname{ker}(f)=\operatorname{Ann}_{R}(V)$.

A module ${ }_{R} M$ is said to have $V$-dom $\operatorname{dim} M \geq 2$ if there exists an exact sequence $0 \rightarrow M \rightarrow X_{0} \rightarrow X_{1}$ such that $X_{0}$ and $X_{1}$ are isomorphic to a direct product of copies of $V$. Since a module ${ }_{R} N$ belongs to $\mathscr{L F}(T)$ if and only if it can be embedded in a direct product of copies of $V$, and a direct product of copies of $V$ is of the form $S(B)$ for a free $E$-module $B_{E}$, it follows that $V$-dom $\operatorname{dim} M \geq 2$ if and only if there exists an exact sequence $0 \rightarrow M \rightarrow S(B) \rightarrow N$, such that $B_{E}$ is a free $E$-module and $N \in \mathfrak{W F}(T)$. For any $B \in \mathbb{N}_{E}$, it can be shown by merely considering a free $E$-resolution of $B$ that $V-\operatorname{dom} \operatorname{dim} S(B) \geq 2$. Recall that ${ }_{R} V$ is said to be balanced if $\phi_{R}: R \rightarrow S T(R)$ is an epimorphism. The next corollary, which is Theorem 5.1 of [11], is a consequence of the preceding remarks and Theorem 4.9.

Corollary (4.10) Let $V$ be a faithful left $R$-module. Then $V$ is balanced if and only if $V$ is of type $F_{b}$ and $V$-dom $\operatorname{dim}{ }_{R} R \geq 2$.

If $P_{R}$ is a right $R$-module, then $P$ is a left $E$-module for the ring $E=\operatorname{End}_{R}(P)$, and the functor $T={ }_{E} P \otimes_{R}-:{ }_{R} \mathbb{M} \rightarrow{ }_{E} \mathbb{M}$ has a right adjoint $S={ }_{R} \operatorname{Hom}_{E}(P,-)$ : $E M \rightarrow{ }_{R}$ M. In this situation, $S T(R)$ is just $\operatorname{End}_{E}(P)$, which can be identified with $\mathrm{Bic}_{R}(P)$. The module $P_{R}$ is said to be of type $F_{t}$ if $\psi_{T(R)}$ is an isomorphism. If $P_{R}$ is flat, then as before, $Q_{T}(R)$ is the ring of quotients determined by $P$, and so by Lemma 4.7 the ring of quotients coincides with $\mathrm{Bic}_{R}(P)$ if and only if $P$ is of type $F_{t}$. Dropping the assumption that $P_{R}$ is flat, if $W$ is a cogenerator in $E^{M}$, let $S(W)=V$. If $B \in E^{M}$, then since $W$ is a cogenerator, $W$-dom $\operatorname{dim} E^{B} \geq 2$, and applying $S$, which preserves direct products, shows that $V$-dom $\operatorname{dim}{ }_{R} S(B) \geq 2$. On the other hand, if $V$-dom $\operatorname{dim} R^{M} \geq 2$, then by definition there exists an exact sequence $0 \rightarrow M \rightarrow S(B) \rightarrow N$, with $B$ isomorphic to a direct product of copies of $W$, and $N$ isomorphic to a direct product of copies of $S(W)$ and therefore in $\mathscr{L F}(T)$. Applying Theorem 4.9 then yields the following result, which is Theorem 5.3 of [11].

Corollary (4.11) Let $P_{R}$ be a faithful right $R$-module, and let $E=$ End $_{R}(P)$. If $W \in E_{E}{ }^{M}$ is a cogenerator, let ${ }_{R} \operatorname{Hom}_{E}(P, W)=V$. Then $P$ is balanced if and only if $P$ is of type $F_{t}$ and $V \cdot \operatorname{dom} \operatorname{dim}_{R} R \geq 2$. 


\section{REFERENCES}

1. Goro Azumaya, Some properties of TTF-classes, Proc. Conf. on Orders, Group Rings, and Related Topics, Lecture Notes in Math, vol. 353, Springer-Verlag, Berlin and New York, 1973.

2. John A. Beachy, Generating and cogenerating structures, Trans. Amer. Math. Soc. 158 (1971), 75-92. MR 44 \#5358.

3. - Cotorsion radicals and projective modules, Bull. Austral. Math. Soc. 5 (1971), 241-253. MR 45 \#1961.

4. Kent R. Fuller, Density and equivalence, (1973) (preprint).

5. A. G. Heinicke, Triples and localizations, Canad. Math. Bull. 41 (1971), 333-339.

6. J. P. Jans, Some aspects of torsion, Pacific J. Math. 15 (1965), 1249-1259. MR 33 \#163.

7. Joachim Lambek, Torsion theories, additive semantics, and rings of quotients, Lecture Notes in Math., vol. 177, Springer-Verlag, Berlin and New York, 1971. MR 44 $\# 1685$.

8. - Localization and completion, J. Pure Appl. Algebra 2 (1972), 343-370.

9. Barry Mitchell, Theory of categories, Pure and Appl. Math., vol. 17, Academic Press, New York, 1965. MR 34 \#2647.

10. Kiiti Morita, Localizations in categories of modules. I, Math. Z. 114 (1970), 121-144. MR $41 \# 8457$.

11. - Flat modules, injective modules and quotient rings, Math. Z. 120 (1971), 25-40. MR $44 \# 4040$.

12. Bruno J. Mueller, The quotient category of a Morita context, (1972) (preprint).

13. Bo Stenström, Rings and modules of quotients, Lecture Notes in Math., vol 237, Springer-Verlag, Berlin and New York, 1971.

14. R. G. Swan, Algebraic K-theory, Lecture Notes in Math., no. 76, Springer-Verlag, Berlin and New York, 1968. MR 39 \#6940.

15. Hiroyuki Tachikawa, On splitting of module categories, Math. Z. 111 (1969), 145150. MR $40 \# 192$.

DEPARTMENT OF MATHEM ATICS, NORTHERN ILLINOIS UNIVERSITY, DEKALB, ILLINOIS 60115

DEPARTMENT OF MATHEMATICS, INDIANA UNIVERSITY, BLOOMINGTON, INDIANA 47401 